\title{
CORRELAÇÕES FENOTÍPICAS, GENOTÍPICAS E DE AMBIENTE EM CLONES DE BATATA-DOCE [Ipomoea batatas (L.) Lam.], RIO LARGO - ALAGOAS
}

\author{
Jair Tenório Cavalcante ${ }^{2 *}$, Paulo Vanderlei Ferreira ${ }^{1}$ \& Lailton Soares ${ }^{1}$ \\ 'Escola Agrícola de Jundiaí, Universidade Federal do Rio Grande do Norte, RN 160, Km 03, Distrito de Jundiaí, CEP: \\ 59280-000, Macaíba, RN. \\ ${ }^{2}$ Centro de Ciências Agrárias, Universidade Federal de Alagoas, 57100-000, Rio Largo, AL. \\ *Autor parar correspondência: Jair Tenório Cavalcante, jairtc@bol.com.br.
}

\begin{abstract}
RESUMO - O experimento foi realizado em Rio Largo - Alagoas, em 1999, objetivando avaliar clones de batatadoce, através de análise de variância, comparação das médias e correlações fenotípicas, genotípicas e de ambiente. Utilizou o delineamento experimental em blocos casualizados, com 14 tratamentos e quatro repetições, analisando as variáveis: rendimento, comprimento, diâmetro médio e número de raízes comerciais, peso da parte aérea, rendimento de raízes não comerciais, resistência à broca do coleto e resistência à ferrugem branca. De um modo geral foram baixas as correlações entre as variáveis, com exceção dos coeficientes de correlações genotípicas. No entanto, houve uma maior contribuição dos fatores genéticos nas correlações. 0 rendimento foi influenciado positivamente tanto pelo diâmetro médio como pelo número de raízes comerciais e o diâmetro médio da raiz foi influenciado negativamente pelo comprimento da mesma. A variável peso da parte aérea não é eficiente na seleção dos clones mais produtivos.
\end{abstract}

Palavras chaves: Ipomoea batatas, correlações, genotípicas, fenotípicas, ambiente.

\section{PHENOTYPIC, GENOTYPIC AND SURROUNDINGS CORRELATIONS IN SWEET POTATOES CLONES (Ipomoea potatoes (L.) Lam.), IN RIO LARGO - ALAGOAS}

\begin{abstract}
The experiment was carried out in Rio Largo - country side of Alagoas/Brazil, in 1999, with the purpose to evaluate sweet potatoes clones throughout analyses of variance, comparison of means and genotypic, phenotypic and surroundings correlations. The experimental design used was the causal blocks with fourteen treatments and four repetitions. The following variables was analysed: yield, length, medium diameter, number of commercial roots, aerial segmental weight, non-commercial roots income, drill of the stem resistance, white rust resistance. In a general way they were low the correlations among the variables and the correlations among the variables, of the coefficients of relationships genotypic were higher; however there was a larger contribution of the genetic factors in the correlations. The revenue was influenced positively so much by the medium diameter as for the number of commercial roots and the medium diameter of the root were influenced negatively by the length of the same. The variable of the aerial segmental weight is not already efficient in the selection of the most productive clones.
\end{abstract}

Key words: Ipomoea batatas. correlations, genopypes, phenotypes, environment.

\section{INTRODUÇÃO}

A batata-doce [Ipomoea batatas (L.) Lam.], é uma das mais importantes hortaliças no Brasil (Miranda et al., 1984), por ser uma excelente fonte de carboidratos e razoável de vitaminas $\mathrm{A}$ e $\mathrm{C}$, é de grande importância social. A planta é rústica, de ampla adaptação, alta tolerância à seca e de fácil cultivo (Silva \& Lopes, 1995). Apresenta custo de produção relativamente baixo, com investimentos mínimos, e de retornos elevados (Miranda et al., 1995). A ocorrência de pragas e doenças, o uso de tecnologia inadequada de produção e a baixa atividade de pesquisa visando o desenvolvimento de novas cultivares implicam baixa produtividade média brasileira, que está em torno de 8,7 t/ha. Em Alagoas, a situação é mais desafiadora, o rendimento médio é de 7,7 tha, em função dos problemas citados acima, principalmente pela 
inexistência de cultivares selecionadas para o cultivo na região (FIBGE, 1999).

0 desenvolvimento de cultivares para as mais diversas condições ambientais é conseguido por meio de pesquisas em melhoramento genético de plantas. Contudo, para aumentar as chances de êxito do programa de melhoramento, bem como possibilitar reduções de tempo, custo e esforços dispendidos, deve-se fazer o uso das correlações fenotípicas, genotípicas e de ambiente entre os caracteres. De acordo com Cruz \& Regazi (1994), o fitomelhorista preocupa-se em aprimorar o material genético não para caracteres isolados, mas para um conjunto destes simultaneamente. Vencovsky (1987), também indica que os estudos das correlações entre caracteres de importância agronômica, aplicamse em trabalhos de melhoramento por servir como base para os melhoristas decidirem sobre 0 material que deve ser selecionado ou descartado. A principal vantagem da estimação de correlações genéticas, em programas de melhoramento, reside na possibilidade de obtenção de ganhos indiretos nos caracteres desejáveis de difícil seleção, devido à baixa herdabilidade ou de problemas de identificação e quantificação (Soares, 1991).

Em programas de melhoramento genético de plantas, é de grande importância o conhecimento da correlação entre caracteres, principalmente se a seleção em um deles apresenta dificuldades, por apresentar-se com baixa herdabilidade e/ou com problemas de medição e identificação. A correlação fenotípica pode ser diretamente mensurada a partir de medidas de dois caracteres de certo número de indivíduos de uma população. Esta correlação tem causas genéticas e ambientais. As correlações genéticas são causadas principalmente por pleiotropia, ligações gênicas, especialmente em populações derivadas de cruzamentos entre linhagens divergentes. 0 ambiente torna-se também causa de correlações quando dois caracteres são influenciados pelas mesmas diferenças de condições ambientais (Cruz \& Regazi, 1994). No contexto apresentado, 0 presente trabalho teve como objetivo avaliar clones de batata-doce desenvolvidos em Alagoas, através de análise de variância, comparações das médias e correlações genotípicas, fenotípicas e de ambiente, nas condições de clima e solo do município de Rio Largo-AL.

\section{MATERIAL E MÉTODOS}

O experimento foi realizado no Centro de Ciências Agrárias, Universidade Federal de Alagoas, localizado no Campus Delza Gitaí, BR 104 Norte, $\mathrm{km} \mathrm{85,} \mathrm{Rio} \mathrm{Largo} \mathrm{-} \mathrm{Alagoas,} \mathrm{no} \mathrm{ano} \mathrm{de} \mathrm{1999.} \mathrm{O}$ solo foi classificado como Latossolo Vermelho. 0 município está situado a uma altitude de $9^{\circ} 27^{\prime} \mathrm{S}$, longitude de $35^{\circ} 27^{\prime} \mathrm{W}$ e uma altitude de $127 \mathrm{~m}$, com temperaturas médias de máxima $29^{\circ} \mathrm{C}$ e mínima de $21^{\circ} \mathrm{C}$, e pluviosidade média anual de $1.267,7 \mathrm{~mm}$. Foram avaliados 14 clones da batata-doce, obtidos a partir de sementes de populações de polinização livre, em novembro de 1997. São eles: CL-01, CL-03, CL-04, CL-05, CL-10, CL-11 e CL-12, provenientes da cultivar Co Copinha; CL-09, proveniente da cultivar Paulistinha Branca; CL-13 e CL-14, provenientes da cultivar Roxa de Rama Fina; CL-02, proveniente da cultivar Co Branca; CL-06, proveniente da cultivar 60 Dias; CL-07, proveniente da cultivar Copinha; e CL08, proveniente da cultivar Pixaim I. O delineamento experimental utilizado foi em blocos casualizados, com 14 tratamentos e quatro repetições. As unidades experimentais foram constituídas por três leiras de 6,0 m de comprimento x 0,3 m de altura cada, com 15 plantas por leira, utilizando-se o espaçamento de $0,80 \mathrm{~m} \times 0,40 \mathrm{~m}$, considerando-se como área útil a fileira central, avaliando-se sete plantas alternadas e competitivas, a partir da segunda.

Após análise de solo, procedeu-se o preparo da área, onde foram efetuadas duas gradagens: uma antes e outra após a aplicação do corretivo. Não foi aplicado fertilizante, visto que o solo apresentou uma boa fertilidade natural e também para melhor caracterizar 0 cultivo na região. Decorridos 30 dias, levantaram-se as leiras através de sulcador tratorizado. Na ocasião do plantio, foram utilizadas ramas novas de até 90 dias, sadias, com 8 a 10 entrenós, dos quais 3 a 4 enterrados no topo da leira. Utilizou-se irrigação por aspersão, devido a ocorrência de veranicos nos primeiros 60 dias após o plantio. As parcelas experimentais foram mantidas livres de ervas daninhas, por meio de capinas à enxada. As pragas e doenças não foram controladas. A partir dos 90 dias após o plantio e até a colheita das raízes tuberosas foram, quinzenalmente observados os danos causados pela ocorrência de pragas e doenças, anotando-se a percentagem de danos, para avaliação do grau de resistência dos referidos clones de batata- 
doce. Aos 130 dias após o plantio, foi efetuada a colheita das raízes tuberosas na leira central. As variáveis relacionadas a seguir referem-se às médias de sete plantas competitivas da leira central de cada parcela experimental: 1 - Rendimento de Raízes Comerciais (RRC): o peso em tha das raízes comerciais (acima de $80 \mathrm{~g}$ ), através de balança de precisão; 2 -Diâmetro Médio da Raiz (DMR): refere-se ao diâmetro, em centímetros, da parte intermediária transversal da raiz, utilizando-se 20 tubérculos tomados aleatoriamente. Sendo a medição feita com a utilização de um paquímetro; 3 - Comprimento da Raiz (CR): refere-se ao comprimento da raiz, em centímetros, utilizando-se os mesmos 20 tubérculos avaliados no DMR. Medição feita através de uma régua; 4 - Número de Raízes Comerciais por Planta (NRC): refere-se a quantidade de raízes comerciais (acima de $80 \mathrm{~g}$ ) por planta; 5 Rendimento de Raízes não Comerciais (RRNC): referese ao peso em t/ha, das raízes não comerciais (de 40 a $80 \mathrm{~g}$ ), através de balança de precisão; 6 - Peso da Parte Aérea da Planta (PPA): refere-se ao peso, em t/ha, da parte aérea das plantas, retirando-se a $0,3 \mathrm{~m}$ do nível do solo, através de balança de precisão; 7 - Resistência à Broca do Coleto (RBC): quinzenalmente foram atribuídas notas de 1 a 5 , à percentagem de plantas atacadas pela Broca do coleto (Megastes pusialis) por parcela experimental. Sendo 1- (0-10\%) Resistente (R);
2 - (11-25\%) Moderadamente Resistente (MR); 3 - (2635\%) Moderadamente Suscetível (MS); 4 - (36-50\%) Suscetível (S) e 5 - (>50\%) Altamente Suscetível (AS). Esta escala foi idealizada por Azevedo et al., 1996; 8 Resistência à Ferrugem Branca (RFB): quinzenalmente foram atribuídas notas de 1 a 5 , à percentagem da área foliar atacada pela Ferrugem branca (Albugo ipomoea - panduratae) nas plantas. Sendo 1- (0-1\%) Resistente (R); 2 - (2-10\%) Moderadamente Resistente (MR); 3 - (11-25\%) Moderadamente Suscetível (MS); 4 - (26$50 \%$ ) Suscetível (S) e 5 - (>50\%) Altamente Suscetível (AS). Esta escala foi idealizada por Peixoto et al. (1989).

Os dados obtidos foram submetidos a análise de variância, comparação das médias e correlações fenotípicas, genotípicas e de ambiente, através do programa computacional Genes: Versão Windows; aplicativo computacional em genética e estatística.

\section{RESULTADOS E DISCUSSÃO}

$\mathrm{Na}$ análise de variância verifica-se a existência de diferenças significativas a $1 \%$ de probabilidade pelo teste $\mathrm{F}$ em todos as variáveis (Tabela 1). Deste modo, pelo menos dois tratamentos (clones) possuem efeitos diferentes sobre a variável analisada, a este nível de probabilidade, com um grau de confiança superior a 99\% de probabilidade.

Tabela 1 - Resumo da análise de variância de oito variáveis avaliadas em 14 clones de batata-doce [lpomoea batatas (L.) Lam.] no delineamento em blocos ao acaso. Rio Largo - Al, 1999

\begin{tabular}{cccccccccc}
\hline & & \multicolumn{7}{c}{ Quadrados Médios } \\
\cline { 3 - 9 } FV & GL & PRC & CR & DMR & PPA & NRC & PRNC & RBC & RFB \\
\hline Blocos & 3 & 18,6995 & 7,4778 & 0,3116 & 0,4490 & 0,6157 & 1,1814 & 1,9762 & 0,0695 \\
& & & & & & & & & \\
Clones & 13 & $64,9676^{* *}$ & $13,0502^{* *}$ & $3,1025^{* *}$ & $1,7548^{* *}$ & $1,0662^{* *}$ & $2,9525^{* *}$ & $3,0659^{* *}$ & $2,6636^{* *}$ \\
& & & & & & & & & \\
Resíduo & 39 & 11,5739 & 1,8254 & 0,2398 & 0,5146 & 0,2763 & 0,5455 & 0,6044 & 0,0318 \\
\hline Média & & 11,46 & 17,38 & 4,78 & 1,55 & 2,12 & 2,05 & 3,61 & 1,34 \\
C.V.(\%) & & 29,69 & 7,77 & 10,25 & 46,39 & 24,74 & 36,08 & 21,55 & 13,55 \\
\hline
\end{tabular}

**: Significativo a $1 \%$ de probabilidade pelo teste $\mathrm{F}$.

1/ PRC: Produtividade de Raízes Comerciais (t/ha); CR: Comprimento da Raiz (cm); DMR: Diâmetro Médio da Raiz (cm); PPA: Peso da Parte Aérea (t/ ha); NRC: Número de Raízes Comerciais (unid.); PRNC: Produtividade de Raízes Não Comerciais (t/ha); RBC: Resistência à Broca do Coleto (nota de 1-5); RFB: Resistência à Ferrugem Branca (nota de 1-5).

A maioria dos coeficientes de variação apresentou estimativas acima de $20 \%$, como os das variáveis PRC, PPA, NRC, PRNC e $\mathrm{RBC}$, enquanto que os demais variaram entre $7,77 \%$ e $13,28 \%$. É comum encontrarem-se valores de coeficiente de variação elevados em variáveis relacionadas a órgãos e/ou estruturas subterrâneas, pois o controle do ambiente é dificultado. Resultados semelhantes foram encontrados por Soares (1991), com batatabaroa (Arracacia xanthorrhiza Bancroft.), cenoura (Daucus carota L.), mandioca (Manihot esculenta 
Crantz), batatinha (Solanum tuberusum L.) e inhame (Colocasia esculenta (L.) Schott.).

Diferenças significativas foram apontadas pelo teste de Tukey a $5 \%$ de probabilidade entre as médias dos 14 clones para cada variável (Tabela 2). Com relação à variável $\mathrm{PRC}, 0$ clone 13 apresentou a maior média em produtividade de raízes comerciais $(19,97$ t/ha) e diferiu dos clones 02, 04, 05, 07, 08, 10 e 11 que produziram em média 8,26 t/ha. Observa-se que os resultados são promissores, visto que Peixoto et al., 1989, avaliaram clones de batata-doce provenientes de sementes botânicas em Formosa - Goias e obtiveram resultados inferiores ao presente trabalho ( clone mais produtivo - BDI 006 - 16,7 t/ha), com ciclo de 146 dias e em condições mais favoráveis, pois foi utilizada adubação mineral. Observa-se também, que apenas dois clones apresentaram produtividades inferiores à média estadual: 0 clone 07 (5,35 tha) e o clone 11 (7,07 tha). Quanto à variável $\mathrm{CR}$, o clone 01 apresentou a maior média de comprimento $(20,78 \mathrm{~cm})$ e diferiu dos clones $03,04,06,07,08,13$, e 14 que apresentaram os menores valores (média de 16,03 cm).

Tabela 2 - Médias de oito variáveis dos 14 clones de batata-doce (Ipomoea batatas (L.) Lam). Rio Largo- Al, 1999.

\begin{tabular}{ccccccccc}
\hline Clones & RRC & C R & DMR & PPA & NRC & PRNC & RBC & RFB \\
\hline CL-01 & $13,52 \mathrm{abcd}$ & $20,78 \mathrm{a}$ & $4,38 \mathrm{cde}$ & $1,88 \mathrm{ab}$ & $2,47 \mathrm{abc}$ & $1,64 \mathrm{bcd}$ & $3,75 \mathrm{abcd}$ & $1,00 \mathrm{~b}$ \\
CL-02 & $8,63 \mathrm{bcd}$ & $19,08 \mathrm{ab}$ & $4,23 \mathrm{de}$ & $3,06 \mathrm{a}$ & $1,75 \mathrm{abc}$ & $3,16 \mathrm{abc}$ & $3,75 \mathrm{abcd}$ & $1,00 \mathrm{~b}$ \\
CL-03 & $14,43 \mathrm{abc}$ & $17,18 \mathrm{bcd}$ & $5,78 \mathrm{~b}$ & $1,13 \mathrm{~b}$ & $1,97 \mathrm{abc}$ & $1,13 \mathrm{~d}$ & $4,00 \mathrm{abc}$ & $3,03 \mathrm{a}$ \\
CL-04 & $10,37 \mathrm{bcd}$ & $16,15 \mathrm{bcd}$ & $4,83 \mathrm{bcde}$ & $1,06 \mathrm{bc}$ & $1,97 \mathrm{abc}$ & $1,31 \mathrm{~cd}$ & $4,00 \mathrm{abc}$ & $3,48 \mathrm{a}$ \\
CL-05 & $7,68 \mathrm{~cd}$ & $17,80 \mathrm{abc}$ & $4,03 \mathrm{e}$ & $0,98 \mathrm{~b}$ & $1,61 \mathrm{bc}$ & $1,69 \mathrm{bcd}$ & $2,50 \mathrm{~cd}$ & $1,00 \mathrm{~b}$ \\
CL-06 & $12,71 \mathrm{abcd}$ & $16,60 \mathrm{bcd}$ & $5,28 \mathrm{bcd}$ & $0,86 \mathrm{~b}$ & $2,47 \mathrm{abc}$ & $1,83 \mathrm{bcd}$ & $4,50 \mathrm{ab}$ & $1,00 \mathrm{~b}$ \\
CL-07 & $5,35 \mathrm{~d}$ & $16,45 \mathrm{bcd}$ & $4,50 \mathrm{cde}$ & $1,19 \mathrm{~b}$ & $1,25 \mathrm{c}$ & $1,39 \mathrm{~cd}$ & $4,75 \mathrm{a}$ & $1,00 \mathrm{~b}$ \\
CL-08 & $9,10 \mathrm{bcd}$ & $17,10 \mathrm{bcd}$ & $4,33 \mathrm{cde}$ & $0,92 \mathrm{~b}$ & $1,82 \mathrm{abc}$ & $3,45 \mathrm{ab}$ & $4,00 \mathrm{abc}$ & $1,00 \mathrm{~b}$ \\
CL-09 & $13,54 \mathrm{abcd}$ & $19,35 \mathrm{ab}$ & $4,05 \mathrm{de}$ & $2,16 \mathrm{ab}$ & $2,89 \mathrm{ab}$ & $2,25 \mathrm{abcd}$ & $2,25 \mathrm{~cd}$ & $1,28 \mathrm{~b}$ \\
CL-10 & $9,65 \mathrm{bcd}$ & $17,48 \mathrm{abc}$ & $4,80 \mathrm{bcde}$ & $0,94 \mathrm{~b}$ & $1,75 \mathrm{abc}$ & $1,45 \mathrm{~cd}$ & $2,75 \mathrm{bcd}$ & $1,03 \mathrm{~b}$ \\
CL-11 & $7,07 \mathrm{~cd}$ & $18,68 \mathrm{ab}$ & $3,68 \mathrm{e}$ & $2,29 \mathrm{ab}$ & $1,82 \mathrm{abc}$ & $3,88 \mathrm{a}$ & $4,50 \mathrm{abc}$ & $1,00 \mathrm{~b}$ \\
CL-12 & $11,43 \mathrm{abcd}$ & $18,00 \mathrm{abc}$ & $4,48 \mathrm{cde}$ & $1,59 \mathrm{ab}$ & $2,36 \mathrm{abc}$ & $1,99 \mathrm{bcd}$ & $4,00 \mathrm{abc}$ & $1,00 \mathrm{~b}$ \\
CL-13 & $19,97 \mathrm{a}$ & $13,78 \mathrm{~cd}$ & $7,05 \mathrm{a}$ & $2,06 \mathrm{ab}$ & $2,65 \mathrm{ab}$ & $1,35 \mathrm{~cd}$ & $2,00 \mathrm{~d}$ & $1,00 \mathrm{~b}$ \\
CL-14 & $17,01 \mathrm{ab}$ & $14,98 \mathrm{~cd}$ & $5,50 \mathrm{bc}$ & $1,54 \mathrm{ab}$ & $3,00 \mathrm{a}$ & $2,15 \mathrm{abcd}$ & $3,75 \mathrm{abcd}$ & $1,00 \mathrm{~b}$ \\
\hline
\end{tabular}

1/ - Médias seguidas pela mesma letra em cada coluna, não diferem entre si, a $5 \%$ de probabilidade, pelo teste de Tukey. ${ }^{1 / R R C: ~ R e n d i m e n t o ~ d e ~ R a i ́ z e s ~}$ Comerciais (t.ha-1); CR: Comprimento da Raiz (cm); DMR: Diâmetro Médio da Raiz (cm); PPA: Peso da Parte área (t.ha-1); NRC: Número de Raízes Comerciais (unidades); RRNC: Rendimento de Raízes não Comerciais (t.ha-1); RBC: Resistência a Broca do Coleto (nota de 1-5); RFB: Resistência a Ferrugem Branca (nota de 1-5)

O clone 13 apresentou o maior diâmetro de raiz $(7,05 \mathrm{~cm})$, para a variável $\mathrm{DMR}$, diferindo estatisticamente de todos os demais. Com relação à variável PPA, o clone dois (02) apresentou o maior peso da parte aérea (3,06 tha), diferindo estatisticamente dos clones 03, 04, 05, 06, 07, 08 e 10 que apresentaram, em média, 1,01 tha. Quanto à variável NRC, 0 clone 14 apresentou o maior número de raízes comerciais $(3,0)$ e diferiu estatisticamente apenas dos clones 05 e 07 que apresentaram, em média, 1,43 unidades.

Com relação à variável PRNC, o clone 11 apresentou a maior produtividade de raízes não comerciais (3,88 t/ha), o que é indesejável, e diferiu estatisticamente dos clones 01, 03, 04, 05, 06, 07, 10, 12 e 13, cuja média foi de 1,53 tha. Resultados semelhantes também foram obtidos por Peixoto et al., (1989), em experimentos realizados em Goiás. Todos os clones produziram mais de uma t/ha de raízes não comerciais. Resende \& Costa (1995), obtiveram com o cultivar Brazilândia Roxa um RRNC de 6,9 t/ ha, resultado este que ultrapassa cerca de $77,84 \% 0$ clone 11. Por outro lado, quando se considera o PRNC em relação a produtividade total de raízes, verifica-se que para o caráter RRNC, o CL 13 foi o que mais se destacou, pois obteve apenas $6,32 \%$, seguido do $\mathrm{CL}$ 03 com 7,23\%; os clones 01, 04, 06, 07, 09, 10, 12 e 14 ficaram entre 10 e 15\%; os demais ficaram acima de 18,04\%. Para à variável RBC, o clone 13 apresentou o menor percentual de plantas atacadas pela broca do coleto, sendo classificado como moderadamente resistente (MR), e diferiu estatisticamente dos clones 03, 04, 06, 07, 08, 11 e 12, que apresentaram os maiores percentuais de ataque desta praga. Quanto à variável RFB, os clones 03 e 04 apresentaram os 
maiores percentuais de sintomas da ferrugem branca, sendo classificados como moderadamente suscetíveis (MS) e diferiram estatisticamente dos demais clones, os que apresentaram um percentual de sintoma da referida doença em torno de $1 \%$, foram classificados como resistentes $(R)$.

$\mathrm{Na}$ Tabela 3, são apresentadas as estimativas dos coeficientes de correlações fenotípica $\left(r_{F}\right)$, genotípica $\left(r_{G}\right)$ e de ambiente $\left(r_{A}\right)$ entre as oito variáveis analisadas em 14 clones de batata-doce. De modo geral, tem-se que são baixas as correlações existentes entre as variáveis, exceto entre as correlações genotípica (-) CR x RRC; fenotípica e genotípica DMR x RRC; ambiente PPA $x$ RRC; fenotípica e genotípica NRC $x$ $\mathrm{RRC}$; fenotípica(-) e genotípica DMR $x$ CR; genotípica NRC x DMR; fenotípica(-) e genotípica RRNC $x$ DMR e genotípica RRNC x PPA. Observa-se que em aproximadamente $80 \%$ dos casos, os coeficientes de correlações genotípicas foram mais elevados do que os das correlações fenotípicas e de ambiente, indicando moderada influência do ambiente na expressão dos caracteres. Resultados semelhantes foram citados por Amaral Jr. (1994), em estudo de acessos de moranga (Cucurbita maxima Duchesne), através da Análise Multivariada e Isozimática da Divergência Genética. A maior magnitude de correlação genotípica, acima de 1,0 , explica-se devido ao fato de se tratar de estruturas ou órgãos subterrâneos, o que dificulta o controle ambiental, de acordo com Soares (1991), ocorre entre as variáveis $\mathrm{RRC}$ e NRC, contrariamante ao observado entre RRC e PPA que apresentaram a menor correlação genotípica (-0,0081). De acordo com Falconer (1987), como as correlações de ambiente foram baixas, tal fato demonstra que as correlações entre as variáveis são pouco influenciadas por variações provocadas pelas condições ambientais.

Tabela 3 - Estimativas dos Coeficientes de Correlações Fenotípicas $\left(r_{F}\right)$, Genotípicas $\left(r_{G}\right)$ e de Ambiente $\left(r_{A}\right)$ entre oito variáveis tomadas duas a duas, avaliadas em 14 clones de batata-doce (Ipomoea batatas). Rio Largo-AL, 1999

\begin{tabular}{|c|c|c|c|c|c|c|c|c|}
\hline Variáveis & $\mathrm{R}$ & CR & DMR & PPA & NRC & RRNC & RBC & RFB \\
\hline & $\mathrm{F}$ & $-0,4132$ & 0,7916 & 0,1188 & 0,8430 & $-0,3573$ & $-0,4373$ & 0,0897 \\
\hline \multirow[t]{2}{*}{ RRC } & G & $-0,5459$ & 0,8583 & $-0,0081$ & 1,0209 & $-0,3946$ & $-0,4688$ & 0,0954 \\
\hline & A & 0,2901 & 0,3765 & 0,5465 & 0,3178 & $-0,1895$ & $-0,3013$ & 0,0807 \\
\hline & $\mathrm{F}$ & & $-0,7670$ & 0,3533 & $-0,1309$ & 0,3373 & 0,0936 & $-0,1531$ \\
\hline \multirow[t]{3}{*}{ CR } & G & & $-0,8513$ & 0,4330 & $-0,1474$ & 0,4174 & 0,1439 & $-0,1748$ \\
\hline & A & & $-0,0831$ & 0,0772 & $-0,0698$ & $-0,0758$ & $-0,1565$ & 0,1960 \\
\hline & $\mathrm{F}$ & & & $-0,1408$ & 0,3984 & $-0,5467$ & $-0,2622$ & 0,2078 \\
\hline \multirow[t]{3}{*}{ DMR } & G & & & $-0,2386$ & 0,5077 & $-0,5967$ & $-0,2658$ & 0,2210 \\
\hline & A & & & 0,3445 & $-0,1509$ & $-0,2441$ & $-0,2706$ & $-0,1067$ \\
\hline & $\mathrm{F}$ & & & & 0,2256 & 0,4706 & $-0,1587$ & $-0,2694$ \\
\hline \multirow[t]{3}{*}{ PPA } & G & & & & 0,3004 & 0,6660 & $-0,0925$ & $-0,3017$ \\
\hline & A & & & & 0,0298 & $-0,1499$ & $-0,3705$ & $-0,2925$ \\
\hline & $\mathrm{F}$ & & & & & $-0,0872$ & $-0,3374$ & $-0,0948$ \\
\hline \multirow[t]{3}{*}{ NRC } & G & & & & & $-0,2125$ & $-0,4327$ & $-0,1123$ \\
\hline & A & & & & & 0,3560 & $-0,0164$ & 0,0224 \\
\hline & $\mathrm{F}$ & & & & & & 0,2489 & $-0,3998$ \\
\hline \multirow[t]{3}{*}{ RRNC } & G & & & & & & 0,2059 & $-0,4406$ \\
\hline & A & & & & & & 0,0923 & $-0,0928$ \\
\hline & $\mathrm{F}$ & & & & & & & 0,1479 \\
\hline \multirow[t]{2}{*}{ RBC } & G & & & & & & & 0,1672 \\
\hline & A & & & & & & & $-0,0224$ \\
\hline
\end{tabular}

1/ RRC: Rendimento de Raízes Comerciais (t/ha); CR: Comprimento da Raiz (cm); DMR: Diâmetro Médio da Raiz (cm); PPA: Peso da Parte Aérea (t/ha); NRC: Número de Raízes Comerciais (unid.); RRNC: Rendimento de Raízes não Comerciais (t/ha); RBC: Resistência à Broca do Coleto (nota de 1-5); RFB: Resistência à Ferrugem Branca (nota de 1-5); r: correlações; F: fenotípicas; G: genotípicas e A: de ambiente.

De maneira geral, das correlações genéticas e ambientais apresentam o mesmo sinal. Em 50\% dos casos, as correlações genotípicas e de ambiente, apresentaram o mesmo sinal, como exemplo: 
DMR $x$ RRC, NRC x RRC, entre outros, indicando que as causas da variação genética e ambiental influenciam as variáveis por meio do mesmo mecanismo fisiológico. Entretanto nos casos onde isto não ocorre, há indicativo das causas de variação genética e ambiental influenciar os caracteres por diferentes mecanismos fisiológicos. Ferreira \& Borém (1996) estudando correlações e análise de trilha, em uma população de milho palha roxa (Zea mays L.), encontraram resultados semelhantes.

Entre os clones analisados, nota-se que a variável RRC correlacionou-se fenotípica e genotipicamente de maneira positiva e com alta magnitude, com o DMR e o NRC, este já esperado. $A$ variável $C R$ correlacionou-se fenotípica e genotipicamente negativo e com alta magnitude com a variável DMR, já com as demais variáveis as correlações foram baixas. Das correlações fenotípicas, $53,58 \%$ são negativas, enquanto que $46,42 \%$ são positivas. No entanto, $85,71 \%$ das correlações fenotípicas são baixas; genotípicas, $57,14 \%$ são negativas, enquanto que $42,86 \%$ são positivas. Também, $75,00 \%$ das correlações genotípicas são baixas; e de ambiente, $57,14 \%$ são negativas, enquanto que $42,86 \%$ são positivas; ainda, $96,42 \%$ das correlações de ambiente são baixas. A avaliação do rendimento e de seus componentes, suas interações com 0 ambiente, juntamente com as correlações entre as variáveis, contribuirão para definir a maneira de agir em um programa de melhoramento genético, podendo-se, desse modo, antecipar a obtenção de resultados e tornar o programa mais objetivo.

\section{CONCLUSÕES}

Existem materiais genéticos distintos $\mathrm{e}$ com potenciais para o cultivo na região, pois, aproximadamente $80 \%$ dos clones avaliados superam o rendimento médio de raízes comerciais de batatadoce do Estado de Alagoas;

A seleção indireta, com base na resposta correlacionada, pode levar a progressos mais rápidos do que a seleção direta do caráter desejado, possibilitando antecipar a obtenção de resultados e tornar o programa mais objetivo.
AMARAL JR, A. T. do. Análise multivariada e isozimática da divergência genética entre acessos de moranga. 1994. Dissertação de Mestrado. Universidade Federal de Viçosa, Viçosa.

AZEVEDO, S. M.; MALUF, W. R.; FREITAS, J. A.; AYRES, F. A. B.; MARTINS, V. S.; MOTA, F. M. F.; SANTOS, E. P.; BRAGA, R.; S.; SILVA, R. M. Avaliação de clones avançados de batata-doce quanto à produtividade, formato e resistência a insetos do solo - UFLA - MG. Revista Horticultura brasileira, 1996. v. 14, no 1, p. 69. (Resumo).

CRUZ, C. D.; REGAZZI, A.J. Métodos biométricos aplicados ao melhoramento genético. Viçosa: U. F. V, Imp. Univ., 1994. 390p.

FALCONER, D. S. Introdução à genética quantitativa. Viçosa: U. F.V., Imp. Univ., 1987. 279p.

FERREIRA, G. A. D.; BORÉM, A. Correlações e análise de trilha em uma população de milho palha roxa (Zea mays L.) - Viçosa: 1996. 13p.

\section{FUNDAÇÃO INSTITUTO BRASILEIRO DE} GEOGRAFIA E ESTATÍSTICA. Anuário estatístico do Brasil. Rio de Janeiro: Secretaria de Planejamento da Presidência da República. 1999. v. 59, p. 3-36.

MIRANDA, J. E. C.; FRANÇA, F. H.; CARRIJO, O. A.; SOUZA, A. F.; PEREIRA, W.; LOPES, C. A.; SILVA, J. B. C. A cultura da batata-doce. Brasília: EMBRAPA. 1995. 94p.

MIRANDA, J. E. C.; FRANÇA, F. H.; CARRIJO, 0. A.; SOUZA, A. F.; AGUILLAR, J. A. E. Cultivo da batata-doce (Ipomoea batatas (L.) Lam) EMBRAPA - CNPH, Brasília. 1984. 8p. (Instruções Técnicas, 7).

PEIXOTO, N.; MIRANDA, J. E. C. de; FILGUEIRA, F. A. R.; CÂMARA, F. L. A. Avaliação de clones de batatadoce em Goiás. Goiânia, EMGOPA-DDT. 1989. 12p. (EMGOPA. Boletim de Pesquisa, 16).

RESENDE, G. M. de; COSTA, N. D. Avaliação de cultivares de batata-doce (Ipomoea batatas (L) 
Lam.). Em condições de sequeiro no Norte de clones com base em procedimentos multivariados Minas Gerais (EMBRAPA / CPATSA, Petrolina PE). e estimativas de parâmetros genéticos. 1991. 73p. In Revista Horticultura brasileira. 1995. v.13, no 1, Dissertação de Mestrado - Universidade Federal de p. 107. Viçosa, Viçosa, MG.

SILVA, J. B. C.; LOPES, C. A. Cultivo da batata-doce. 3. Ed. Brasília: EMBRAPA. 1995. 18p. (Instruções Técnicas de CNPHortalilças -7).

VENCOVSKY, R. Herança quantitativa. In: PATERNIANI, E. Melhoramento e produção de milho no Brasil. Piracicaba, ESALQ, 1987. 409p.

SOARES, L. Melhoramento de batata-baroa (Arracacia xanthorrhiza Bancroft). II - Divergência genética entre 